\title{
HALL CURRENT EFFECTS ON FREE CONVECTION MHD FLOW PAST A POROUS PLATE
}

\author{
P.V.Satya Narayana ${ }^{1}$, G. Ramireddy ${ }^{2}$ and S. Venkataramana ${ }^{2}$ \\ ${ }^{1}$ Department of Mathematics, VIT University \\ Vellore-632 014, Tamilnadu, India \\ Phone: +91 9789574488, Fax: +0416-2243091 \\ E-Mail: pvsatya8@yahoo.co.in,psatya@vit.ac.in \\ ${ }^{2}$ Department of Mathematics, S.V.University \\ Tirupati, A.P, India
}

\begin{abstract}
Heat and mass transfer along a vertical porous plate under the combined buoyancy force effects of thermal and species diffusion is investigated in the presence of a transversely applied uniform magnetic field and the Hall currents are taken into account. The governing fundamental equations on the assumption of a small magnetic Reynolds number are approximated by a system of non-linear ordinary differential equations, which are integrated by fourth-order Runge-Kutta method. Velocity, temperature and concentration are shown on graphs. The numerical values of the local shear stress, the local Nusselt number $N u$ and the local Sherwood number $S h$ are entered in tables. The effects of the magnetic parameter, Hall parameter and the relative buoyancy force effect between species and thermal diffusion on the velocity, temperature and concentration are discussed. The results are compared with those known from the literature.
\end{abstract}

Keywords: Hall current, MHD, free convection, mass transfer, porous medium.

\section{INTRODUCTION}

Hydromagnetic transport in porous media has received considerable attention owing to applications in materials processing, geophysics, astrophysical flows, etc. Magnetic fields induce many complex phenomena in an electrically conducting flow regime, including Hall currents, ion-slip effects (at higher strength magnetic fields), Joule (Ohmic) heating, Alfven waves in plasma flows, etc. Such effects can have a considerable influence on heat transfer and flow dynamics. For example, in ionized gases with low density subjected to a strong magnetic field, the electrical conductivity perpendicular to the magnetic field is lowered owing to free spiralling of electrons and ions about the magnetic lines of force prior to collisions; a current is thereby induced which is mutually perpendicular to both electrical and magnetic fields, constituting the Hall current effect. Katagiri (1969) has studied the effects of Hall current on the magnetohydrodynamic boundary layer flow past a semi-infinite plate. Pop and Soundalgekar (1974) and Gupta (1975) have investigated the effects of Hall current on the steady hydromagnetic flow in an incompressible viscous fluid. Hossain and Rashid (1987) discussed the effects of Hall current on unsteady free convection flow along a porous plate in the presence of foreign gases such as $\mathrm{H}_{2}, \mathrm{CO}_{2}$ and $\mathrm{NH}_{3}$ subjected to a transpiration velocity inversely proportional to the square root of time. Pop and Wattanabe (1994) considered the Hall effect on magnetohydrodynamic free convection 
about a semi-infinite vertical plate and solved the problem numerically by employing a difference-differential method in combination with Simpson's rule. Aboeldahab and Elbarbary (2001) discussed heat and mass transfer along a vertical plate under the combined buoyancy force effects of thermal and species diffusion in the presence of transversely applied magnetic field and taking Hall effect into account. The system of non-linear equations is solved by using Runge-Kutta methods. Chaudhary and Kumar Jha (2008) studied the MHD heat and mass transfer along a vertical plate immersed in a porous medium. Hossain (1986), Hossain and Mohammad (1988), Attia and SyedAhmed (2004), Attia (2008), Attia and Aboul-Hassan (2003) and Rana et al. (2007) studied the Hall effects. However, Aboeldahab (2001) studied the Hall current effects on MHD free convection flow past a semi-infinite vertical plate with radiation. Therefore, the objective of the present investigation is to study the steady magnetohydrodynamic free convection viscous incompressible fluid flow past a semi infinite vertical porous plate with mass transfer under strong external magnetic field.

\section{FORMULATION AND SOLUTION OF THE PROBLEM}

We study the steady magnetohydrodynamic free convection viscous incompressible fluid flow past a semi-infinite vertical porous plate with mass transfer. Here the $\mathrm{x}$-axis is taken to be along the porous plate in the vertically upward direction. The y-axis is taken as being normal to the plate and a strong magnetic field $\mathrm{B}_{0}$ is considered along the $\mathrm{y}-$ axis (Figure 1). The temperature and the species concentration at the plate are $\mathrm{T}_{\mathrm{w}}\left(\neq \mathrm{T}_{\infty}\right)$ and $\mathrm{C}_{\mathrm{w}}\left(\neq \mathrm{C}_{\infty}\right), \mathrm{T}_{\infty}$ and $\mathrm{C}_{\infty}$ being the temperature and species concentration of the free stream. The generalized Ohm's law including Hall current is given in the form given by Marg (1964).

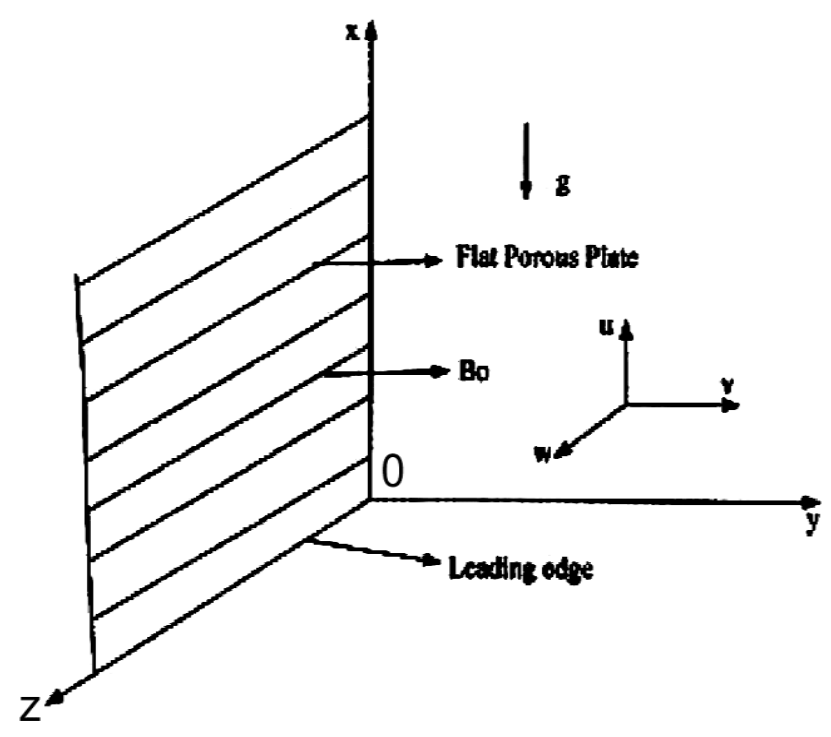

Figure 1. Sketch of the physical model

In the absence of an externally applied electric field and with negligible effects of polarization of the ionized gas, we also assume that $E=0$ (Meyer, 1958). The effect of Hall currents gives rise to a force in the z-direction, which includes a cross flow in that direction, and hence the flow becomes three dimensional. To simplify the problem, we assume that there is no variation of flow, temperature and concentration quantities in 
the z-direction. It is also assumed, a valid assumption on the laboratory scale, that the induced magnetic field is negligible. Since the velocity of the fluid is low, the viscous dissipative heat is assumed to be negligible. The equation of conservation of electric charge $\nabla J=J_{\mathrm{y}}=$ constant. This constant is zero since $J_{\mathrm{y}}=0$ at the plate, which is electrically non-conducting. Thus $J_{\mathrm{y}}=0$ everywhere in the flow. Under these assumptions the boundary layer free convection flow with mass transfer and generalized Ohm's law is governed by the following system of equations (1) - (5):

$$
\begin{gathered}
\frac{\partial u}{\partial x}+\frac{\partial v}{\partial y}=0 \\
u \frac{\partial u}{\partial x}+v \frac{\partial u}{\partial y}=v \frac{\partial^{2} u}{\partial y^{2}}+g \beta\left(T-T_{\infty}\right)+g \beta^{*}\left(C-C_{\infty}\right)-\frac{B_{0} J_{z}}{\rho}-\frac{v}{K} u \\
u \frac{\partial w}{\partial x}+v \frac{\partial w}{\partial y}=v \frac{\partial^{2} w}{\partial y^{2}}+\frac{B_{0}}{\rho} J_{x}-\frac{v}{K} w \\
u \frac{\partial T}{\partial x}+v \frac{\partial T}{\partial y}=\alpha \frac{\partial^{2} T}{\partial y^{2}} \\
u \frac{\partial C}{\partial x}+v \frac{\partial C}{\partial y}=D \frac{\partial^{2} C}{\partial y^{2}}
\end{gathered}
$$

where $J_{x}=\frac{\sigma B_{0}}{\left(1+\mathrm{m}^{2}\right)}(\mathrm{m} u-w)$ and $J_{z}=\frac{\sigma B_{0}}{\left(1+\mathrm{m}^{2}\right)}(u+\mathrm{m} w)$

The appropriate boundary conditions of equations (1) - (5) are

$$
\begin{aligned}
& \mathrm{u}=0, \quad \mathrm{v}=0 \quad \mathrm{w}=0, \quad \mathrm{~T}=\mathrm{T}_{\mathrm{w}} \quad \mathrm{C}=\mathrm{C}_{\mathrm{w}} \quad \text { at } \mathrm{y}=0 \\
& \mathrm{u}=0, \quad \mathrm{v}=0 \quad \mathrm{w}=0, \quad \mathrm{~T}=\mathrm{T}_{\infty} \quad \mathrm{C}=\mathrm{C}_{\infty} \quad \text { as } \mathrm{y} \rightarrow \infty
\end{aligned}
$$

Proceeding with the analysis, we now define the similarity variables as follows:

$$
\begin{aligned}
& \eta=y x^{-\frac{1}{4}}\left(\frac{G r}{4 L^{3}}\right)^{\frac{1}{4}}, \Psi=4 v\left(\frac{G r}{4 L^{3}}\right)^{\frac{1}{2}} x^{\frac{3}{4}} f(\zeta, \eta), \zeta=\left(\frac{\mathrm{x}}{\mathrm{L}}\right)^{\frac{1}{2}}, \\
& \operatorname{Pr}=\left(\frac{v}{\alpha}\right) \text { Prandtl number, } G r=\frac{g \beta L^{3}}{v^{2}}\left(T_{w}-T_{\infty}\right) \text { Grashof number } \\
& G c=\frac{g \beta^{*} L^{3}}{v^{2}}\left(C_{w}-C_{\infty}\right) \text { Modified Grashof number, } S c=\frac{v}{D} \text { Schmidt number } \\
& M=\frac{\sigma \beta_{0}^{2} L^{2}}{\rho v G r^{\frac{1}{2}}} \text { Magnetic parameter, } \quad \mathrm{m}=\frac{\sigma B_{0}}{e n_{e}} \quad \text { Hall parameter } \\
& w=2 v\left(\frac{G r}{L^{3}}\right)^{\frac{1}{2}} x^{\frac{1}{2}} g(\zeta, \eta) \quad, \quad \theta=\frac{T-T_{\infty}}{T_{w}-T_{\infty}} \quad, \quad \phi=\frac{C-C_{\infty}}{C_{w}-C_{\infty}}
\end{aligned}
$$




$$
e=\frac{G c}{G r}, \quad p=\frac{2 L^{2}}{K G r^{\frac{1}{2}}}
$$

The continuity equation is now satisfied by

$$
\begin{aligned}
& u=\frac{\partial \Psi}{\partial y} \\
& v=-\frac{\partial \Psi}{\partial x}
\end{aligned}
$$

Using equations (7) and (8), we may show that

$$
\begin{array}{r}
u=2 v\left(\frac{G r}{L^{3}}\right)^{\frac{1}{2}} x^{\frac{1}{2}} f^{\prime}(\zeta, \eta) \\
v=-v\left(\frac{G r}{L^{3}}\right)^{\frac{1}{2}} x^{-\frac{1}{4}}\left(3 f+2 \zeta \frac{\partial f}{\partial \zeta}-\eta f^{\prime}\right)
\end{array}
$$

The prime indicates the differentiation with respect to $\eta$. In view of equations (7) - (9) we can show that equations $(2)-(5)$ reduce to

$$
\begin{gathered}
f^{\prime \prime \prime}+3 f f^{\prime \prime}-2\left(f^{\prime}\right)^{2}+2 \zeta\left(f^{\prime \prime} \frac{\partial f}{\partial \zeta}-f^{\prime} \frac{\partial^{2} f}{\partial x \partial \zeta}\right)+\theta+e \phi-\frac{2 \mathrm{~m} \zeta}{1+\mathrm{m}^{2}}\left(f^{\prime}+\mathrm{m} g\right)-p \zeta f^{\prime}=0 \\
g^{\prime \prime}+3 f g^{\prime}-2 g f^{\prime}+2 \zeta\left(g^{\prime} \frac{\partial f}{\partial \zeta}-\frac{\partial g}{\partial \zeta}\right)-\frac{2 \zeta M}{1+\mathrm{m}^{2}}\left(g-\mathrm{m} f^{\prime}\right)-p \zeta g=0 \\
\frac{1}{\operatorname{Pr}} \theta^{\prime \prime}+3 f \theta^{\prime}+2 \zeta\left(\theta^{\prime} \frac{\partial f}{\partial \zeta}-f^{\prime} \frac{\partial g}{\partial \zeta}\right)=0 \\
\frac{1}{S c} \phi^{\prime \prime}+3 f \phi^{\prime}+2 \zeta\left(\phi^{\prime} \frac{\partial f}{\partial \zeta}-f^{\prime} \frac{\partial \phi}{\partial \zeta}\right)=0
\end{gathered}
$$

In view of equation (9) the boundary condition (6) reduces to

$$
\begin{array}{r}
f^{\prime}(\zeta, 0)=0, f^{\prime}(\zeta, \infty)=0,3 f(\zeta, 0)+2 \zeta \frac{\partial f(\zeta, 0)}{\partial \zeta}=0, g(\zeta, 0)=0 \\
g(\zeta, \infty)=0, \theta(\zeta, 0)=1, \theta(\zeta, \infty)=, 0, \phi(\zeta, 0)=1 \quad \phi(\zeta, \infty)=0
\end{array}
$$

In free convection problems the Grashof number may be interpreted physically as a dimensionless group representing the ratio of the buoyancy forces due to temperature differences to the viscous forces. Similarly, in free convection and mass transfer flow systems, the modified Grashof number Gc can be interpreted as a dimensionless group representing the ratio of the buoyancy forces due to concentration differences to the viscous forces. So, the dimensionless parameter $e$ is a measure of the ratio of the buoyant forces due to concentration differences to the buoyant forces due to temperature differences and plays an important role in the free convection and mass transfer flow systems. 
The governing boundary layer Eq. (10)- Eq. (14) is approximated by a system of non-linear ordinary differential equations replacing the derivatives with respect to $\eta$ by two point backward finite differences with step size 0.01 . These equations are integrated by a fourth order Runge-Kutta method. The major physical quantities of interest are the local Shear stress $\tau_{\mathrm{x}}$ and $\tau_{\mathrm{z}}$, the local Nusselt number $N u$ and the local Sherwood number $S h$, which are defined, respectively by

$$
\begin{array}{cc}
\tau_{x}=-\left.\mu \frac{\partial u}{\partial y}\right|_{y=0}, & \tau_{\mathrm{z}}=-\left.\mu \frac{\partial \mathrm{w}}{\partial \mathrm{y}}\right|_{\mathrm{y}=0} \\
N u=\left.\frac{-\alpha}{\left(T_{w}-T_{\infty}\right)} \frac{\partial T}{\partial y}\right|_{y=0}, & S h=\left.\frac{-D}{\left(C_{w}-C_{\infty}\right)} \frac{\partial C}{\partial y}\right|_{y=0}
\end{array}
$$

Table 1. Comparison of present results with those of Ali (1995) with different values of Pr for Nusselt number With $M=m=K=e=S c=0$.

\begin{tabular}{llll}
\hline & $\operatorname{Pr}=0.7$ & $\operatorname{Pr}=1.0$ & $\operatorname{Pr}=10.0$ \\
\hline Ali & -0.45255 & -0.59988 & -2.29589 \\
Present work & -0.45308 & -0.59822 & -2.30321 \\
\hline
\end{tabular}

Table 2. Variation of $f^{\prime \prime}, g^{\prime}, \theta^{\prime}, \phi^{\prime}$ at the plate surface with $m=1, e=0.1, K=1$ for different magnetic parameters.

\begin{tabular}{ccccc}
\hline$M$ & $f^{\prime \prime}$ & $g^{\prime}$ & $\theta^{\prime}$ & $\phi^{\prime}$ \\
\hline 0 & 0.6002 & 0 & 0.4 & 0.3421 \\
0.25 & 0.5621 & 0.0012 & 0.4052 & 0.3753 \\
0.50 & 0.5379 & 0.0193 & 0.4276 & 0.4039 \\
0.75 & 0.5101 & 0.0299 & 0.4297 & 0.4272 \\
\hline
\end{tabular}

Table 3. Variation of $\mathrm{f}^{\prime \prime}, \mathrm{g}^{\prime}, \theta^{\prime}, \phi^{\prime}$ at the plate surface with $\mathrm{M}=0.5, e=0.1, \mathrm{~K}=1$ for different Hall parameters.

\begin{tabular}{lllll}
\hline$m$ & $\mathrm{f}^{\prime \prime}$ & $\mathrm{g}^{\prime}$ & $\theta^{\prime}$ & $\phi^{\prime}$ \\
\hline 0 & 0.4728 & 0 & 0.4172 & 0.4268 \\
0.5 & 0.4927 & 0.0127 & 0.4149 & 0.4158 \\
1 & 0.5277 & 0.0221 & 0.4104 & 0.4054 \\
1.5 & 0.535 & 0.022 & 0.4088 & 0.3939 \\
2 & 0.569 & 0.0013 & 0.4048 & 0.3857 \\
3 & 0.5869 & 0.0012 & 0.4023 & 0.3547 \\
$\infty$ & 0.6000 & 0 & 0.4001 & 0.3421 \\
\hline
\end{tabular}

Table 4. Variation of $f^{\prime \prime}, g^{\prime}, \theta^{\prime}, \phi^{\prime}$ at the plate surface with $M=0.5, m=1 K=1$ for 
different $e$ values.

\begin{tabular}{lllll}
\hline$e$ & $\mathrm{f}^{\prime \prime}$ & $\mathrm{g}^{\prime}$ & $\theta^{\prime}$ & $\phi^{\prime}$ \\
\hline 0 & 0.4858 & 0.0222 & 0.3998 & 0.3565 \\
0.1 & 0.5277 & 0.0232 & 0.4104 & 0.3782 \\
0.2 & 0.5779 & 0.024 & 0.4194 & 0.4054 \\
0.3 & 0.6032 & 0.0244 & 0.4284 & 0.4124 \\
\hline
\end{tabular}

Table 1 shows a comparison of the present results for the Nusselt number with Ali (1995) for various values of Prandtl number $\operatorname{Pr}$ with $\mathrm{M}=m=\mathrm{K}=\mathrm{e}=\mathrm{Sc}=0$. The results are found to be in good agreement. In Tables 2-4, we show the effect of the magnetic, Hall, and $e$ parameters on the functions $\mathrm{f}^{\prime \prime}, \mathrm{g}^{\prime}, \theta^{\prime}$ and $\phi^{\prime}$ at the plate surfaces respectively. From the tables we note that the wall shear stress along the plate $f^{\prime \prime}$ decreases, while the shear stress across the plate $\mathrm{g}^{\prime}$ increases with $\mathrm{M}$ increases. On the other hand, $\mathrm{f}^{\prime \prime}$ increases with $m$ increase but g' increases monotonically when $m$ increases to 1 and decreases for values of $m$ greater than 1. The local Nusselt number and local Sherwood number decrease with increasing magnetic parameter $\mathrm{M}$, and $e$ increases. In the case when $m=0$, i.e., in the absence of Hall currents, the flow becomes two-dimensional with the velocity components in $\mathrm{x}$ and $\mathrm{y}$ directions only, i.e., $\mathrm{g}$ vanishes. Also, the $\mathrm{z}$ component of the shear force $\mathrm{g}^{\prime}$ at the plate vanishes identically (see Table 2).

\section{RESULTS AND DISCUSSION}

The present work generalized the problem of free convection flow of an electrically conducting, viscous, incompressible fluid near a semi-infinite vertical porous plate. The fluid motion is subjected to an external strong magnetic field normal to the plate. Hall current effects are included and mass transfer is taken into consideration. After a certain transformation, numerical solution has been obtained by a fourth order Runge-Kutta method. Results are obtained for various values of magnetic parameter M, Hall parameter $m$, relative buoyancy force parameter $e$, and permeability parameter $\mathrm{K}$.

The results of the numerical computations are displayed in the following section. For the primary velocity $f^{\prime}$, secondary velocity $\mathrm{g}$, temperature $\theta$ and concentration $\Phi$ respectively for $S c=0.6, P r=0.72$, are drawn against $\eta$ for different values of $M, m, e$, and $K$. Figures $2-5$ show typical profiles for the fluid tangential velocity $f^{\prime}$, the lateral velocity $g$, temperature $\theta$ and the concentration $\Phi$ for different values of the magnetic parameter respectively. Application of a magnetic field to an electrically conducting fluid produces a drag-like force called the Lorentz force. This force causes reduction in the fluid velocity in the boundary layer which, in turn, reduces the rate of heat and mass convection in the flow and this appears when increasing the fluid temperature and concentration. These behaviours are clearly illustrated in Figures 2, 4 and 5. In the absence of the magnetic field, there is no lateral velocity $(\mathrm{g}=0)$ and as $\mathrm{M}$ increases (consequently $\mathrm{m}$ increases), a cross flow in the lateral direction is greatly induced due to the Hall effect. This is clearly depicted in Figure 3. 


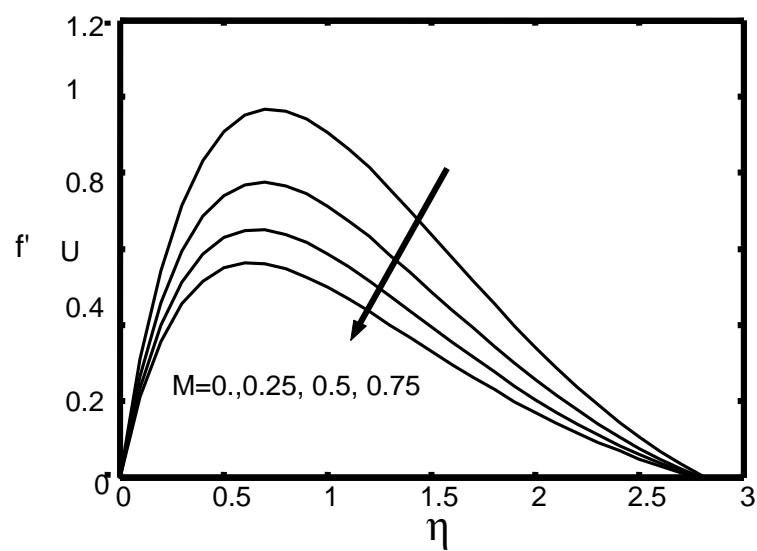

Figure 2. Velocity profiles along the plate $\mathrm{f}^{\prime}$ for different $\mathrm{M}$ values

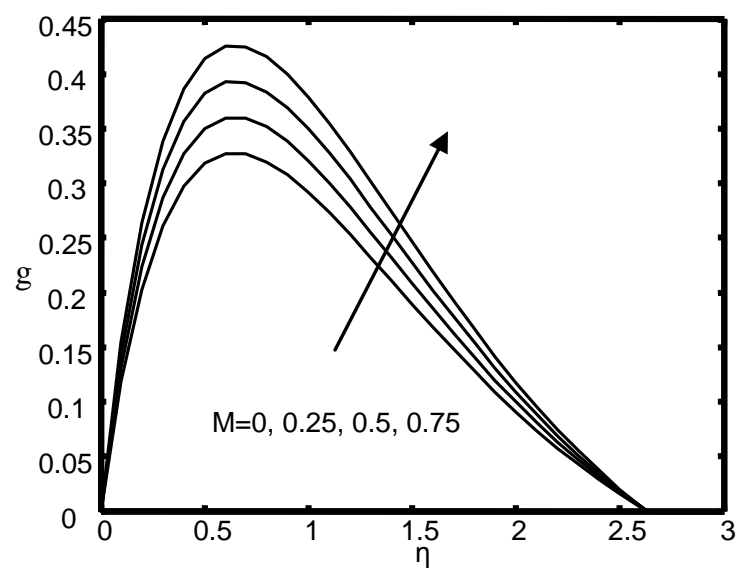

Figure 3. Velocity profiles across the plate $\mathrm{g}$ for different $\mathrm{M}$ values

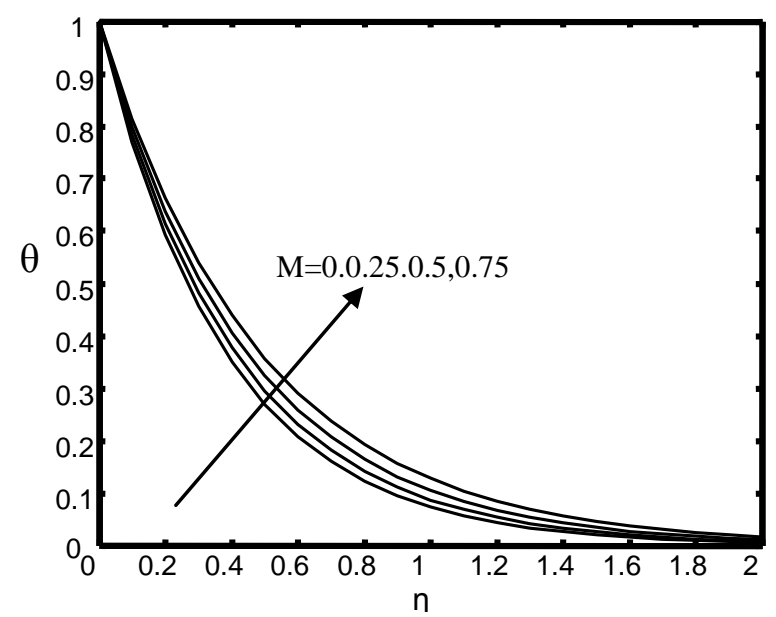

Figure 4. Temperature profiles $\theta$ for different $M$ values 


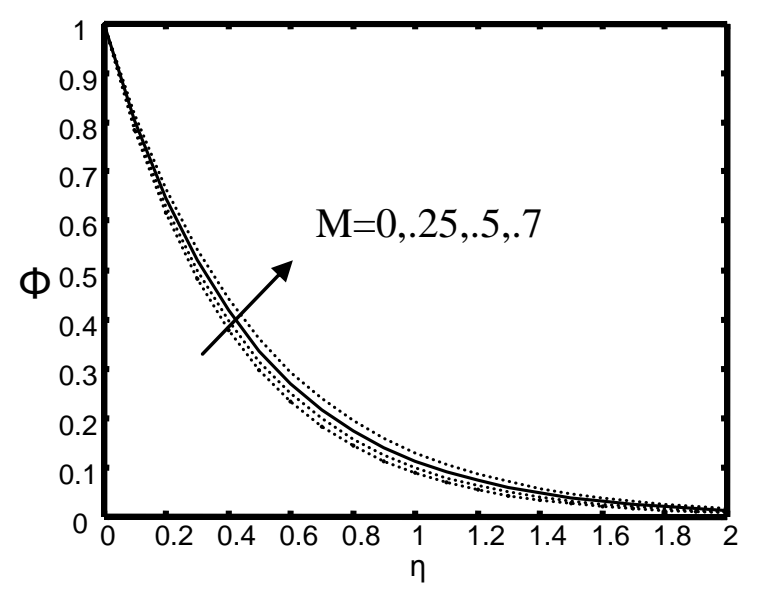

Figure 5. Concentration distribution against $\eta$ for different $M$ values

Figures 6-9 show the $f^{\prime}, g, \theta$ and $\Phi$ profiles for $\mathrm{M}=0.5, \mathrm{e}=0.1, \operatorname{Pr}=0.72 . \mathrm{Sc}=0.6$, and $K=1$ against $\eta$ for different values of the Hall parameter $m$. Further, Figures 6-9 illustrate that the tangential velocity $f^{\prime}$ and lateral velocity increase while the temperature $\theta$ and the concentration $\Phi$ decrease as the Hall parameter $m$ increases, since the magnetic damping on $f^{\prime}$ decreases as $m$ increases, and the magnetic field has a propelling effect on $f^{\prime}$. Also, it is shown that the $f^{\prime}, \theta$ and $\Phi$ profiles approach their classical values when the Hall parameter $m$ increases to 1 , since the magnetic force terms approach zero value for very large values of $m$.

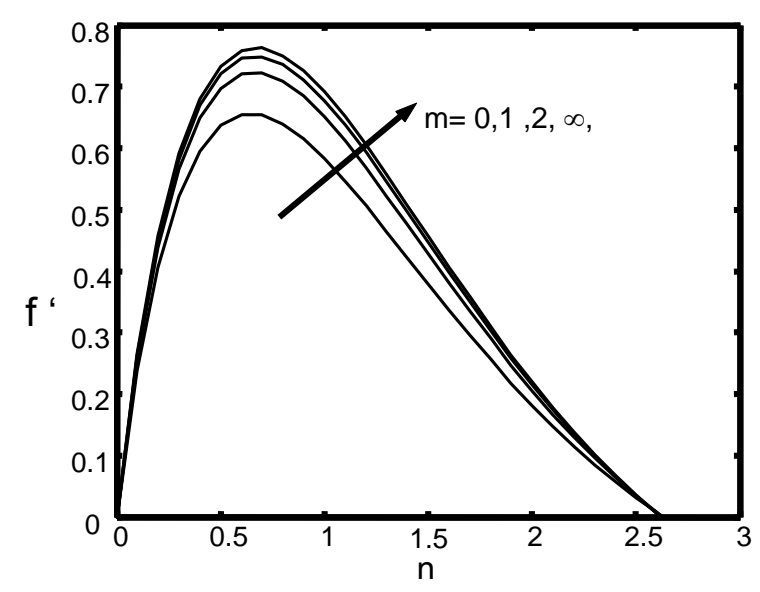

Figure 6. Velocity profile for different values of $m$

Figures 10-13 show typical profiles for the fluid tangential velocity $f^{\prime}$ the lateral velocity g, temperature $\theta$ and the concentration $\Phi$ for different values of ( $\mathrm{e}=0,0.1,0.2$ ) respectively, taking $\mathrm{Sc}=0.6, \operatorname{Pr}=0.72, m=1, \mathrm{~K}=1$ and $m=0.5$. From Figures 10 and 11 , it is observed that $f^{\prime}$ and $g$ increase up to the crossover point after that the variation is reversed. Figures 12 and 13 illustrate that the temperature and concentration are found to decrease with the increase of $\eta$ until it vanishes at $\eta=3$. But both the values decrease for any non-zero fixed value of $\eta$ with the increase of $e$. In Figures 14, the velocity profiles $f^{\prime}$ and $g$ are plotted against $\eta$ for different values of $K(=1,2,3)$ taking $S c=0.6$, $\operatorname{Pr}=0.72, m=1, M=0.5$, and $e=0.1$ respectively. For all the values of $K$ considered, $f^{\prime}$ 
is found to decrease with the increase of $K$ and $g^{\prime}$ is found to increase with the increase of $K$.

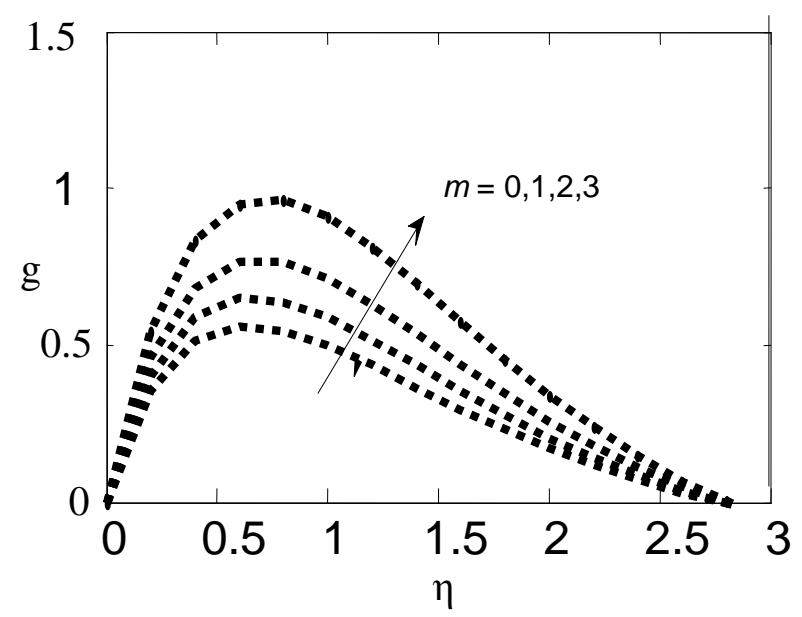

Figure 7. Velocity profile for different values of $m$

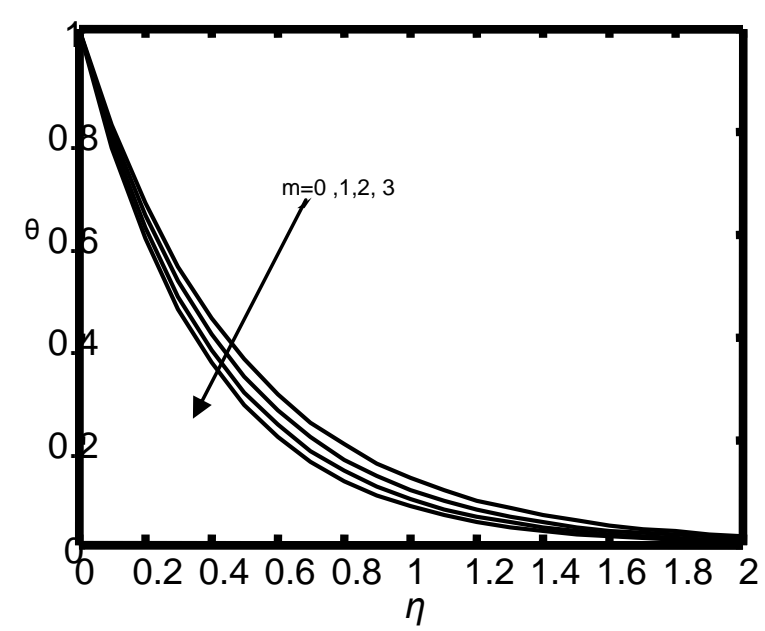

Figure 8. Temperature distribution against $\eta$ for different $m$ values

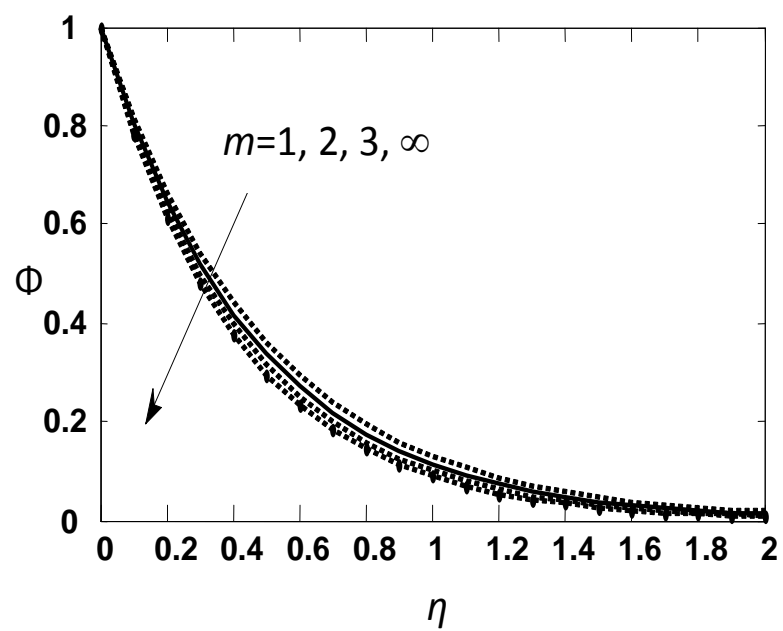

Figure 9. Concentration distribution against $\eta$ for different $m$ values 


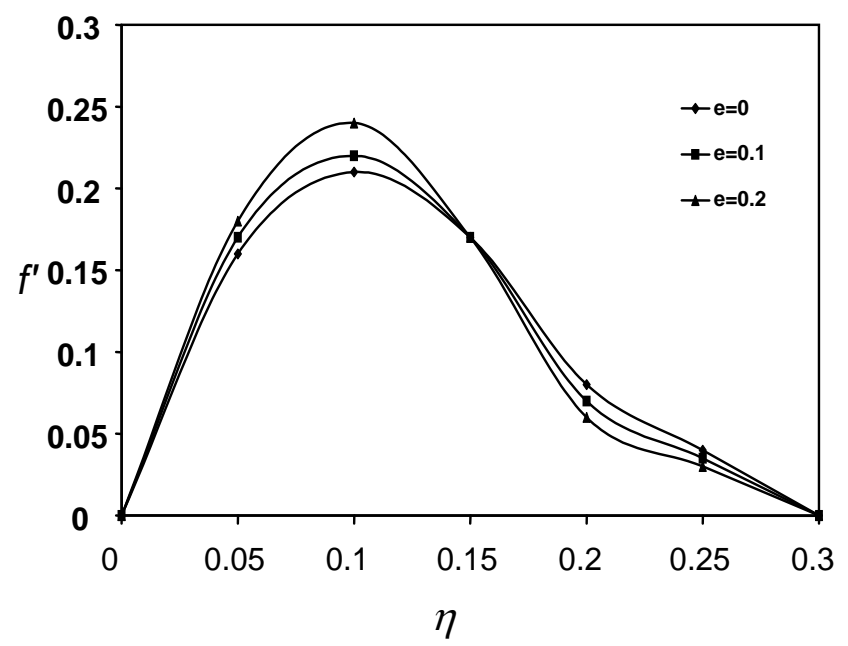

Figure 10. Velocity profiles along the plate $f^{\prime}$ for various $e$ values.

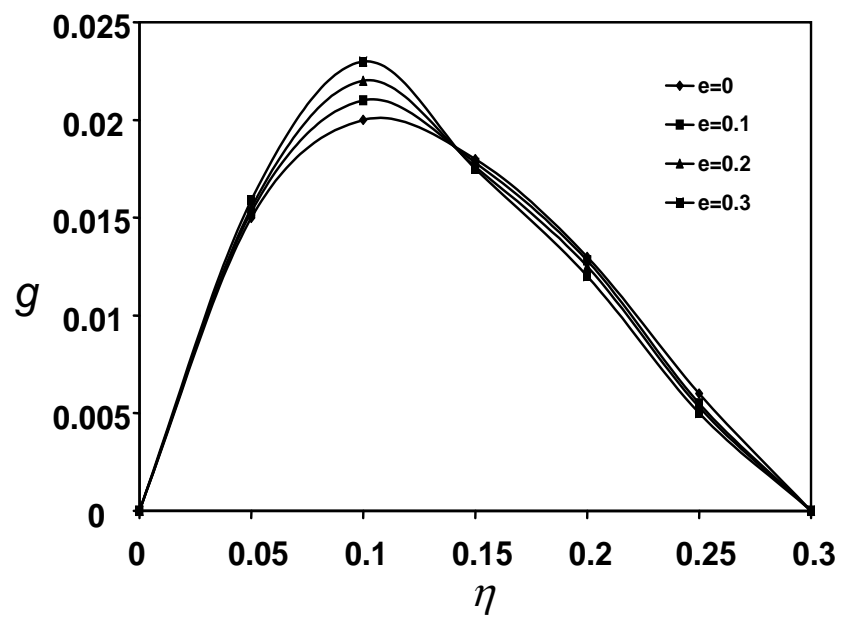

Figure 11. Velocity profiles along the plate $\mathrm{g}$ for different $e$ values

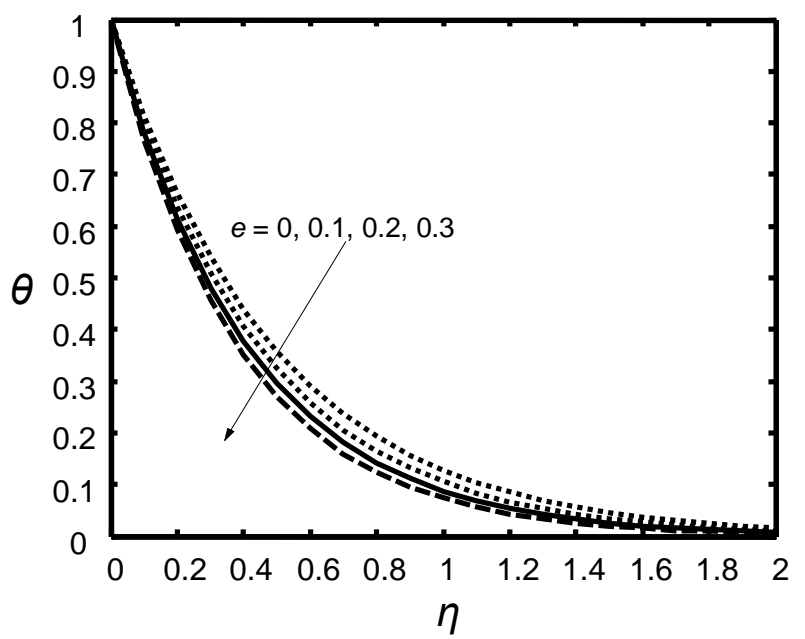

Figure 12. Temperature distribution against $\eta$ for different $e$ values 


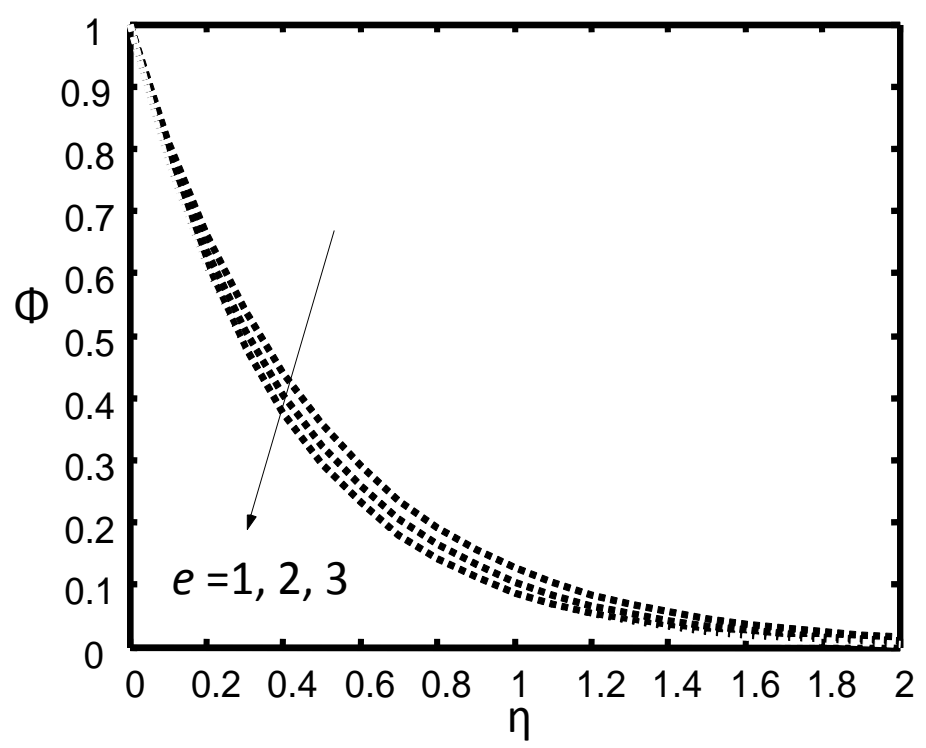

Figure 13. Concentration distribution against $\eta$ for different $e$ values

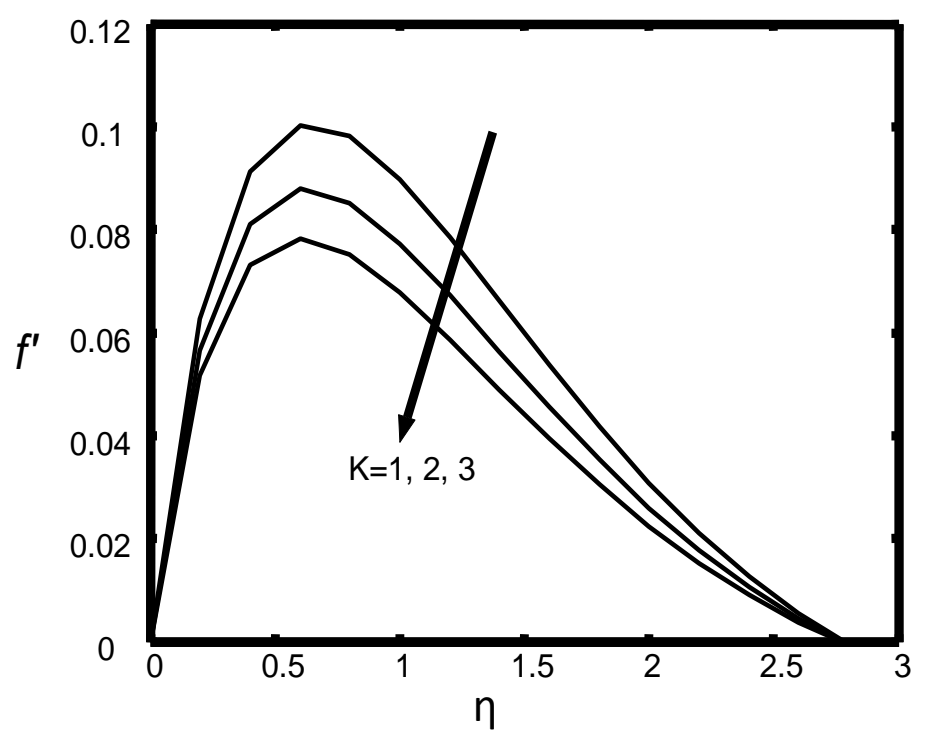

Figure 14 . Velocity profiles across the plate $g$ for different $K$ values

Figures 15 and 16 illustrate the behaviour velocity and temperature for different values of the Prandtl number. The numerical results show that the effect of increasing the values of the Prandtl number results in a decreasing velocity. From Figure 16, it is observed that an increase in the Prandtl number results in a decrease of the thermal boundary layer thickness and in general lower average temperature within the boundary layer. The reason is that smaller values of $\mathrm{Pr}$ are equivalent to an increase in the thermal conductivity of the fluid and therefore heat is able to diffuse away from the heated surface more rapidly for higher values of $\mathrm{Pr}$. 


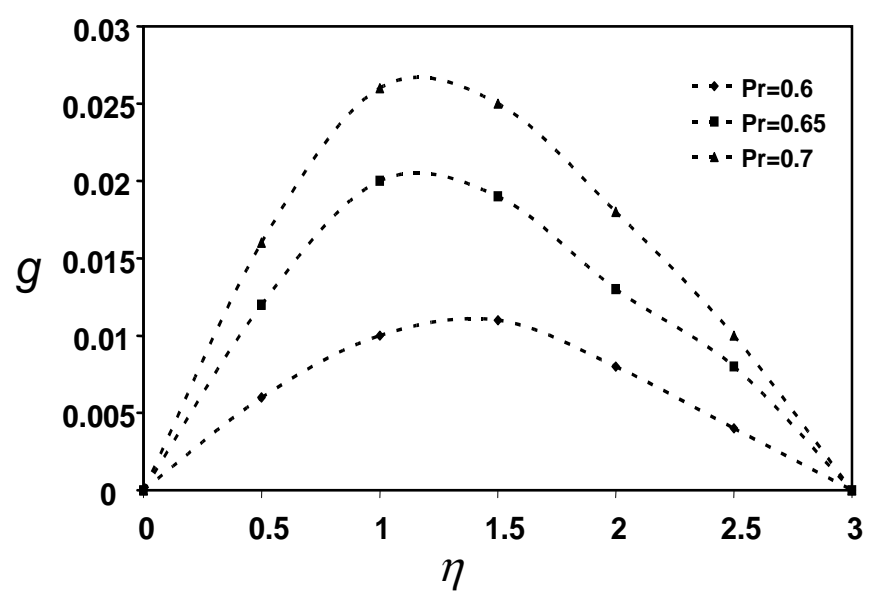

Figure 15 . Velocity profiles along the plate $g$ for different $\operatorname{Pr}$ values

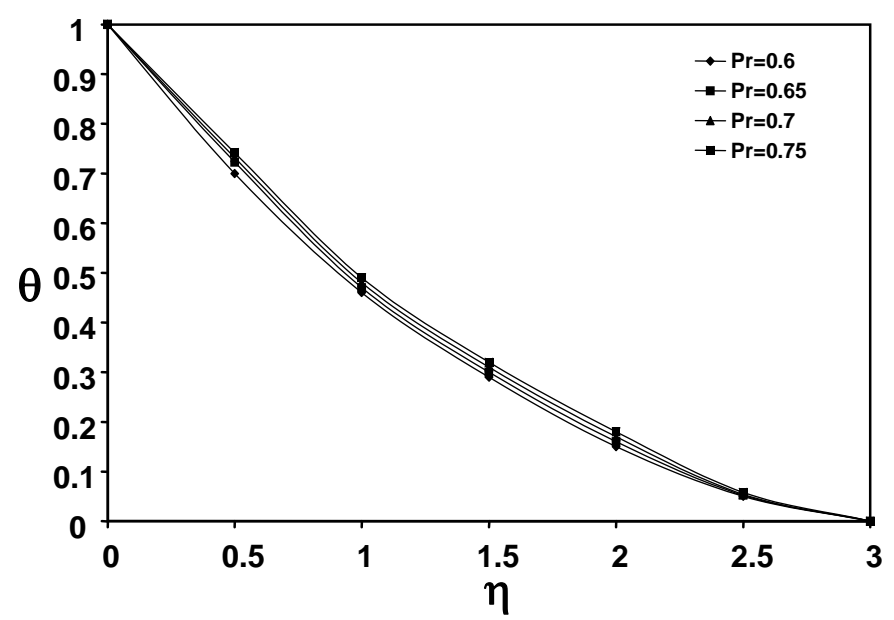

Figure 16. Temperature profiles $\theta$ for different $\operatorname{Pr}$ values

\section{CONCLUSIONS}

The above analysis brings out the following results of physical interest on the velocity, temperature and concentration distribution of the flow field.

1. The magnetic parameter $M$ retards the velocity of the flow field at all points due to the magnetic pull of the Lorentz force acting on the flow field.

2. The effect of Hall parameter $m$ is to increase the velocity of the flow field at all points.

3. The effect of porosity parameter $\mathrm{K}$ is to enhance the velocity of the flow field at all points.

4. The Prandtl number Pr reduces the temperature of the flow field at all points. The higher the Prandtl number, the sharper is the reduction in temperature of the flow field.

5. The concentration distribution of the flow field decreases at all points as the Hall parameter $m$ increases. This means the heavier diffusing species have a greater retarding effect on the concentration distribution of the flow field. 


\section{ACKNOWLEDGEMENTS}

The authors sincerely thank Prof. S. Sreenadh (Dept. of Mathematics, S. V. University, Tirupati- 517502) for useful discussions.

\section{REFERENCES}

Aboeldahab, E.M. 2001. Hall effects on magnetohydrodynamic free convective flow at astretching surface with a uniform free stream. Physica Scripta, 63: 29-35.

Aboeldahab, E.M. and Elbarbary, E.M.E. 2001. Hall current effect on magnetohydrodynamic free convection flow past a semi-infinite vertical plate with mass transfer. International Journal of Engineering Science, 39: 16411652.

Ali, M.E. 1995. On thermal boundary layer on a power-law stretched surface with suction or injection. International Journal of Heat and Fluid Flow, 16: 280-290.

Attia, H.A. 2008. Effect of Hall current on transient hydromagnetic Couette-Poiseuille flow of a viscoelastic fluid with heat transfer. Applied Mathematical Modelling, 32(4): 375-388.

Attia, H.A.and Syed-Ahmed, M.E. 2004. Hall effect on unsteady MHD coutte flow and heat transfer of a Bingham fluid with suction and injection. Applied Mathematical Modelling, 28:1027-1045.

Attia, H.A. and Syed-Ahmed, M.E. 2004. Hall effect on unsteady MHD Couette flow and heat transfer of a Bingham fluid with suction and injection. Applied Mathematical Modelling, 28: 1027-1045.

Attia, H.A. and Aboul-Hassan, A.L. 2003. The effect of variable properties on the unsteady Hartman flow with heat transfer considering the Hall effect. Applied Mathematical Modelling, 27:551-563.

Gupta, A.S. 1975. Hydromagnetic flow past a porous flat plate with Hall effects. Acta Mechanica, 22: 281-287.

Hossain, M.A. and Mohammad, K. 1988. Effect of Hall current on hydromagnetic free convection flow near an accelerated porous plate. Japanese Journal of Applied Physics, 27(8): 1531-1535.

Hossain, M.A. 1986. Effect of Hall current on unsteady hydromagnetic free convection flow near an infinite vertical porous plate. Japanese Journal of Applied Physics, 55(7): 2183-2190.

Hossain, M.A. and Rashid, R.I.M.I. 1987. Hall effect on hydromagnetic free convection flow along a porous flat plate with mass transfer. Japanese Journal of Applied Physics, 56: 97-104.

Katagiri, M. 1969. The effect of Hall currents on the magnetohydrodynamic boundary layer flow past a semi-infinite flat plate. Japanese Journal of Applied Physics, 27: 1051- 1059.

Mary, R.F. 1964. The influence of electric and magnetic fields on heat transfer to electrically conducting fluid, in: Advances in Heat Transfer. New York: Academic Press, pp. 286-354.

Meyer, R.C. 1958. On reducing aerodynamic heat-transfer rates by magnetohydrodynamic techniques. J. Aerospace Science, 25: 516-566.

Pop, I. and Soundalgekar, V.M. 1974. Effects of Hall currents on hydro-magnetic flow near a porous plate. Acta Mechanica, 20: 315-318.

Pop, I. and Wattanabe, T. 1994. Hall effect on magnetohydrodynamic free convection 
about a semi-infinite vertical flat plate. International Journal of Engineering Science, 32: 1903-1911.

Rana, M.A., Siddiqui, A.M. and Qamar, R. 2007. Hall effects on hydromagnetic flow of an Oldroyd 6-constant fluid between concentric cylinders. Chaos Soliton Fractals, 39:204-213.

Chaudhary R.C. and Kumar Jha A. 2008. Heat and mass transfer in elastico-viscous fluid past an impulsively started infinite vertical plate with hall effect. Latin American Applied Research, 38:17-26

\section{NOMENCLATURE}

$\mathrm{u}, \mathrm{v}, \mathrm{w}$ velocity components along the $(\mathrm{x}, \mathrm{y}, \mathrm{z})$ axes respectively.

e charge of electron

B magnetic induction vector which has connection with the intensity of the magnetic field vector

C dimensionless concentration

D mass diffusion coefficient $\mathrm{m}^{2} \cdot \mathrm{s}^{-1}$

E intensity vector of the electric field

$J \quad$ electric current density vector

Gc mass Grashof number

$\mathrm{Gr}$ thermal Grashof number

g acceleration due to gravity $\mathrm{m} . \mathrm{s}^{-2}$

$\mathrm{K}$ porosity parameter

Pr Prandtl number

M magnetic parameter

Sc Schmidt number

$\mathrm{T}$ temperature of the fluid near the plate

$\mathrm{u} \quad$ velocity of the fluid in the $\mathrm{x}$-direction $\mathrm{m} . \mathrm{s}^{-1}$

U dimensionless velocity

Sh local Sherwood number

$\mathrm{Nu}$ local Nusselt number

$\mathrm{n}_{\mathrm{e}} \quad$ the number density of electron

\section{Greek symbols}

$\beta \quad$ volumetric coefficient of thermal expansion $\mathrm{K}^{-1}$

$\beta^{*} \quad$ volumetric coefficient of expansion with concentration $\mathrm{K}^{-1}$

$\mu \quad$ coefficient of viscosity Pa.s

$v \quad$ kinematic viscosity $\mathrm{m}^{2} \cdot \mathrm{s}^{-1}$

$\rho \quad$ density of the fluid kg.m ${ }^{-3}$

$\tau$ dimensionless skin-friction

$\theta$ dimensionless temperature

$\eta \quad$ similarity parameter

$\alpha \quad$ thermal conductivity $\mathrm{J} \cdot \mathrm{m}^{-1} \cdot \mathrm{K}^{-1}$

$\psi \quad$ the stream functions.

$\sigma \quad$ electrical conductivity

$\mu_{\mathrm{e}} \quad$ magnetic permeability 